University of Wollongong

Research Online

Australian Institute for Innovative Materials -

Papers

Australian Institute for Innovative Materials

$1-1-2010$

\title{
Magnetic properties of La doped Bi2FeMnO6 ceramic and film
}

Hongyang Zhao

National Institute Materials Science, Japan

Hideo Kimura

National Institute For Materials Science

Zhenxiang Cheng

University of Wollongong, cheng@uow.edu.au

Xiaolin Wang

University of Wollongong, xiaolin@uow.edu.au

Kiyoshi Ozawa

National Institute for Materials Science, ozawa@uow.edu.au

See next page for additional authors

Follow this and additional works at: https://ro.uow.edu.au/aiimpapers

Part of the Engineering Commons, and the Physical Sciences and Mathematics Commons

Research Online is the open access institutional repository for the University of Wollongong. For further information contact the UOW Library: research-pubs@uow.edu.au 


\title{
Magnetic properties of La doped Bi2FeMnO6 ceramic and film
}

\author{
Abstract \\ We present a comprehensive study of the magnetic properties for La doped $\mathrm{Bi}_{2} \mathrm{FeMnO}_{6}$ (LBFM) ceramic \\ and film which was first deposited on (100) $\mathrm{SrTiO}_{3}$ substrate by pulsed laser deposition method. The \\ LBFM film is antiferromagnetic or weak ferrimagnetic at room temperature and it shows clear magnetic \\ anisotropy. But it is not sure whether the spin-glass behavior is present in LBFM film because of the \\ influence from substrate. Compared to the film, LBFM ceramic shows different magnetic properties. It \\ has a lower TN and all the observations indicate that LBFM ceramic shows spin-glass behavior.

\section{Keywords} \\ Magnetic, properties, doped, Bi2FeMnO6, ceramic, film \\ Disciplines \\ Engineering | Physical Sciences and Mathematics

\section{Publication Details} \\ Zhao, H, Kimura, H, Cheng, Z, Wang, X, Ozawa, K \& Nishida, T (2010), Magnetic properties of La doped \\ Bi2FeMnO6 ceramic and film, Journal of Applied Physics, 108(9), pp. 093903-1-093903-4.
}

\section{Authors}

Hongyang Zhao, Hideo Kimura, Zhenxiang Cheng, Xiaolin Wang, Kiyoshi Ozawa, and Takashi Nishida 


\title{
Magnetic properties of La doped $\mathrm{Bi}_{2} \mathrm{FeMnO}_{6}$ ceramic and film
}

\author{
Hongyang Zhao, ${ }^{1, a)}$ Hideo Kimura, ${ }^{1, b)}$ Zhenxiang Cheng, ${ }^{2}$ Xiaolin Wang, ${ }^{2}$ \\ Kiyoshi Ozawa, ${ }^{1}$ and Takashi Nishida ${ }^{3}$ \\ ${ }^{1}$ National Institute for Materials Science, Sengen 1-2-1, Tsukuba 305-0047, Japan \\ ${ }^{2}$ Institute for Superconducting and Electronics Materials, University of Wollongong, Innovation Campus, \\ Fairy Meadow, New South Wales 2519, Australia \\ ${ }^{3}$ Graduate School of Materials Science, Nara Institute of Science and Technology, 8916-5 Takayama-Cho, \\ Ikoma, Nara 630-0101, Japan
}

(Received 9 June 2010; accepted 15 September 2010; published online 2 November 2010)

\begin{abstract}
We present a comprehensive study of the magnetic properties for $\mathrm{La}$ doped $\mathrm{Bi}_{2} \mathrm{FeMnO}_{6}(\mathrm{LBFM})$ ceramic and film which was first deposited on (100) $\mathrm{SrTiO}_{3}$ substrate by pulsed laser deposition method. The LBFM film is antiferromagnetic or weak ferrimagnetic at room temperature and it shows clear magnetic anisotropy. But it is not sure whether the spin-glass behavior is present in LBFM film because of the influence from substrate. Compared to the film, LBFM ceramic shows different magnetic properties. It has a lower $\mathrm{T}_{\mathrm{N}}$ and all the observations indicate that LBFM ceramic shows spin-glass behavior. (C) 2010 American Institute of Physics. [doi:10.1063/1.3503397]
\end{abstract}

\section{INTRODUCTION}

In recent years, double perovskites of $\mathrm{A}_{2} \mathrm{BB}^{\prime} \mathrm{O}_{6}(\mathrm{~A}=\mathrm{La}$, $\mathrm{Bi}$, etc.; $\mathrm{B}$ or $\mathrm{B}^{\prime}=$ transition metal such as $\mathrm{Ni}, \mathrm{Mn}, \mathrm{Fe}$, and Co) have gained much interests. ${ }^{1-3}$ According to Goodenough-Kanamori's rules, the superexchange magnetic interaction between $\mathrm{B}$ and $\mathrm{B}^{\prime}$, with and without $\mathrm{e}_{\mathrm{g}}$ electrons, may lead to ferromagnetic oxides through $\mathrm{B}-\mathrm{O}-\mathrm{B}^{\prime}-\mathrm{O}-\mathrm{B}$ ordering. ${ }^{4-6}$ Increasing interests are focused on their attractive behaviors which are related to the coexistence of different competing factors of spin, charge, or orbital and lattice degrees of freedom, phase, mixed-valence and so on. ${ }^{7-9}$ In addition, they exhibit multifunctional behaviors, which make them promising candidates for spintronic applications. Recently, pulsed laser deposition (PLD) of epitaxial $\mathrm{Bi}_{2} \mathrm{FeMnO}_{6}$ films has also been reported. ${ }^{10,11}$ The stabilization of single-phase Bi-based perovskites are difficult because of their tendency of multiphase formation and the high volatility of bismuth. ${ }^{12}$ Stabilization can be facilitated by a partial replacement of $\mathrm{Bi}^{3+}$ cations by La cations. ${ }^{13-16} \mathrm{In}$ addition, $\mathrm{LaMn}_{1-\mathrm{x}} \mathrm{Fe}_{\mathrm{x}} \mathrm{O}_{3}$ including $\mathrm{La}_{2} \mathrm{FeMnO}_{6}$ has been also reported to be an interesting mixed-valence manganites with perovskite structure. ${ }^{7,17,18}$ Therefore, La was chosen to substitute $\mathrm{Bi}$ in $\mathrm{Bi}_{2} \mathrm{FeMnO}_{6}$ to stabilization the phase.

Because the structure of films deposited at different conditions is still difficult to confirm, there will be always some difference in electric and magnetic properties between bulk and thin film samples. ${ }^{19}$ The structure and properties of the films were found to depend on the substrate type, film thickness, process condition during deposition, $\mathrm{B} / \mathrm{B}^{\prime}$ ordering and so on. In such a case, both La doped $\mathrm{Bi}_{2} \mathrm{FeMnO}_{6}$ (LBFM) ceramic and thin films were studied in the present paper.

\section{EXPERIMENTAL DETAILS}

Polycrystalline 20 mol \% LBFM ceramics were synthesized by conventional solid state method as the target. LBFM

\footnotetext{
${ }^{a)}$ Electronic mail: zhao.hongyang@nims.go.jp.

${ }^{b)}$ Electronic mail: kimura.hideo@nims.go.jp.
}

films were deposited on (100) $\mathrm{SrTiO}_{3}$ substrate using a PLD system at $650{ }^{\circ} \mathrm{C}$ with 500-600 mTorr dynamic oxygen. The film was annealed at the same condition for $10 \mathrm{~min}$ and then cooled to room temperature rapidly. The phases of the target and film were determined by x-ray diffraction (XRD) using $\mathrm{Cu} K \alpha$ radiation. Magnetic properties were measured using the commercial Quantum Design superconducting quantum interference device magnetometer (MPMS).

\section{EXPERIMENTAL RESULTS AND DISCUSSION}

As shown in Fig. 1, the LBFM diffraction peaks of (100), (200), and (300) were observed in the XRD pattern. It indicates the epitaxial growth of LBFM film on (100) $\mathrm{SrTiO}_{3}$ (STO) substrate. There is no traceable impurity in the film which is believed to have a bulklike cubic structure. But there are unavoidable impurities of bismuth oxides in LBFM ceramics, which make the ceramic have worse crystalline quality than LBFM film.

As shown in Fig. 2(a), the magnetization hysteresis loops of LBFM film were measured at 5, 20, 50, 100, and $300 \mathrm{~K}$. The inset is the magnification of hysteresis loops at $300 \mathrm{~K}$. There is no change in the loop width below $20 \mathrm{~K}$, but at higher temperature the loop width decreased rapidly as the increase in temperature. The M-H loops show small hyster-

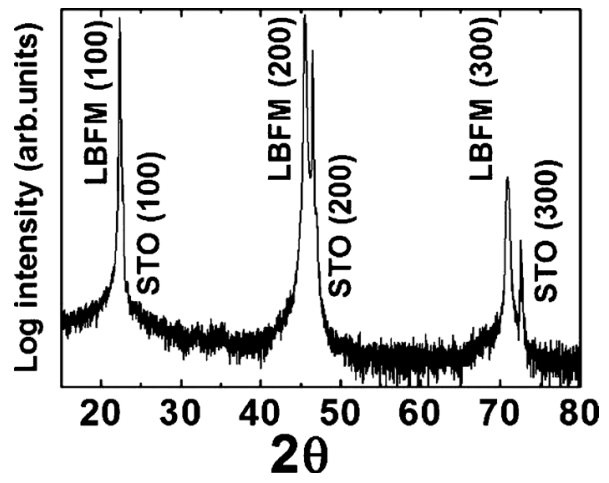

FIG. 1. XRD pattern for LBFM film fabricated on (100) STO. 

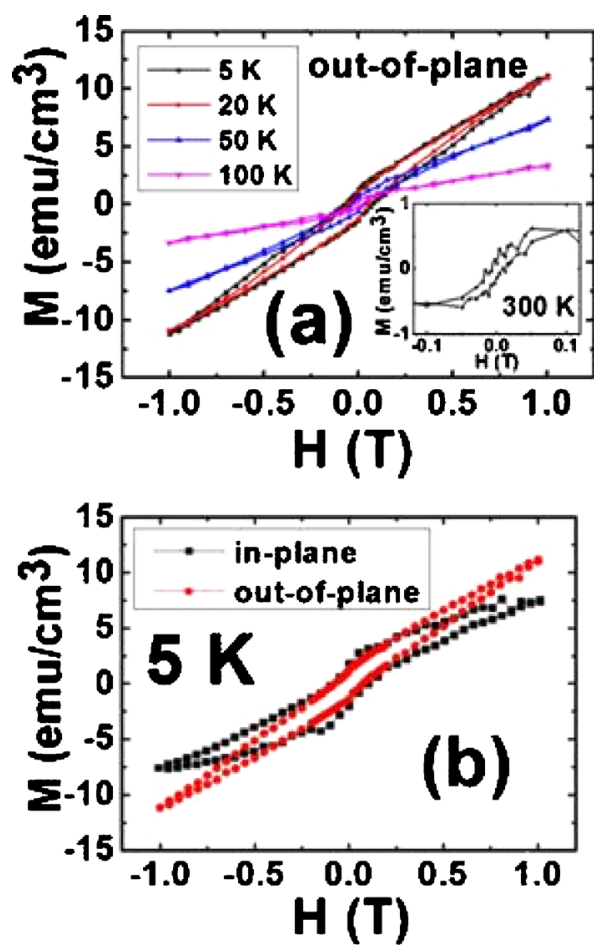

FIG. 2. (Color online) Magnetization hysteresis loops of LBFM film (a) measured at various temperatures and the inset shows magnification of the hysteresis loops at $300 \mathrm{~K}$; (b) measured with magnetic field applied parallel and perpendicular to the sample plane.

esis with small magnetization values which increases linearly with magnetic field and do not saturate at high magnetic field. This is a strong indication that our samples are antiferromagnetic or weak ferrimagnetic (ferromagnetic plus antiferromagnetic configuration) at room temperature. Figure 2(b) shows the in-plane and out-of-plane magnetic field dependence of magnetization measured at $5 \mathrm{~K}$. The film shows anisotropy and out-of-plane is the easy magnetization direction. ${ }^{11,20}$ The out-of-plane zero-field-cooled (ZFC) and field-cooled (FC) magnetization curves measured between 5 and $350 \mathrm{~K}$ were shown in Fig. 3. A spin-glasslike behavior was occurred at $18 \mathrm{~K}$ at $1 \mathrm{kOe}$ applied field. This behavior is similar to $\mathrm{Bi}_{2} \mathrm{FeMnO}_{6}$ film. ${ }^{11}$ It was not overwhelmed at 10 kOe as shown in Fig. 3 as an inset but the peak temperature shifts from 18 to $10 \mathrm{~K}$. As we know, the ac magnetization measurement is very useful for the investigation of the spin

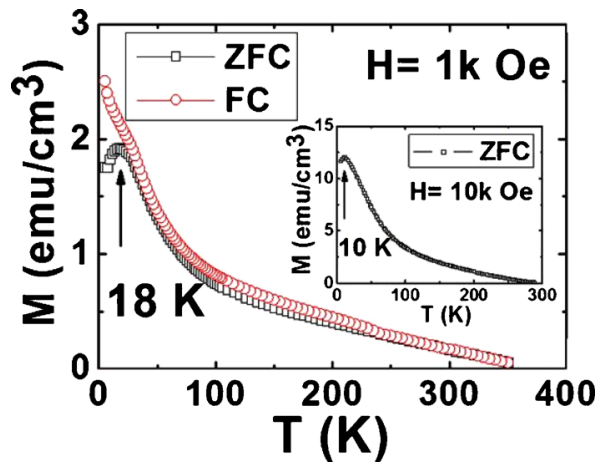

FIG. 3. (Color online) Temperature dependence of ZFC and FC magnetizations of LBFM film recorded at $1 \mathrm{kOe}$. The inset shows $\mathrm{ZFC}$ results at 10 kOe.

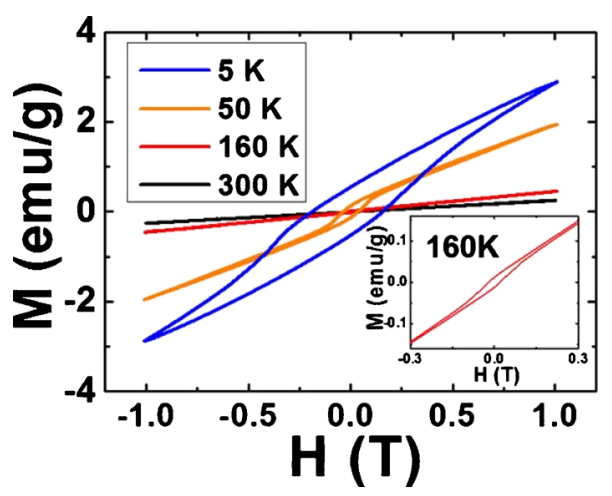

FIG. 4. (Color online) Magnetization hysteresis loops of LBFM ceramic measured at various temperatures. The inset shows the amplification of the loop at $160 \mathrm{~K}$.

glass behavior. We have performed the frequency dependent measurement of the magnetization but there are many noises because of the influences of the substrate. The results are not shown because they are useless for the analysis of spin-glass. In such a case, whether the phenomena in the LBFM film can be classified as a classical spin-glass still remains open.

Figure 4 shows the magnetization hysteresis loops of LBFM ceramic measured at various temperatures. The sample shows unsaturated magnetization for an applied magnetic field up to $1 \mathrm{~T}$. The magnetizations at $1 \mathrm{~T}$ for LBFM ceramic are $2.88,1.94,0.46$, and $0.26 \mathrm{emu} / \mathrm{g}$, corresponding to the temperature of $5 \mathrm{~K}, 50 \mathrm{~K}, 160 \mathrm{~K}$, and $300 \mathrm{~K}$, respectively. The inset shows the amplification of the loop at 160 $\mathrm{K}$, a very small magnetic hysteresis was observed. While the hysteresis disappears at $300 \mathrm{~K}$ that is different from the LBFM film [shown in Fig. 2(a) inset]. Because the structure and properties of the LBFM films can vary depending on the substrate type, film thickness, and deposition conditions.

As shown in Fig. 5, ZFC and FC curves of LBFM ceramic were recorded at different applied field of $500 \mathrm{Oe}, 1$ kOe, $5 \mathrm{kOe}$, and $10 \mathrm{kOe}$. Similar to $\mathrm{Bi}_{2} \mathrm{FeMnO}_{6}$ film ${ }^{11}$ and LBFM film, a spin-glasslike behavior was also observed. The ZFC-peak was not suppressed as the field reached $10 \mathrm{kOe}$ and it shows field dependence. It is difficult to obtain the exact transition temperature. However, it is clear that the ZFC-peak temperature gradually decreases as the increase in the applied field. The high temperature (5-350 K) ZFC and

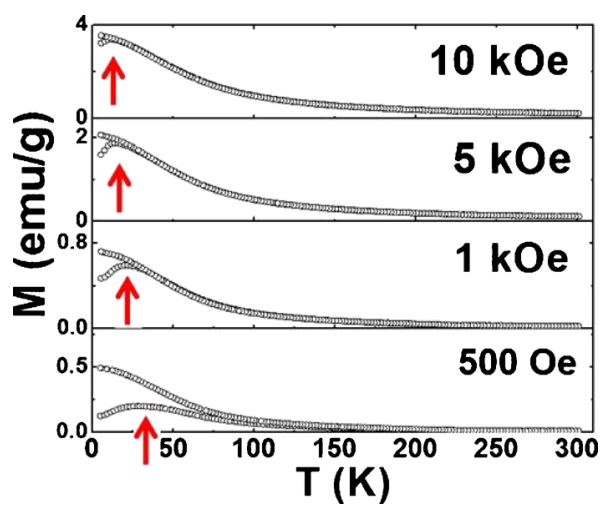

FIG. 5. (Color online) ZFC and FC magnetizations of LBFM ceramic recorded at different applied field of $500 \mathrm{Oe}, 1 \mathrm{kOe}, 5 \mathrm{kOe}$, and $10 \mathrm{kOe}$. 


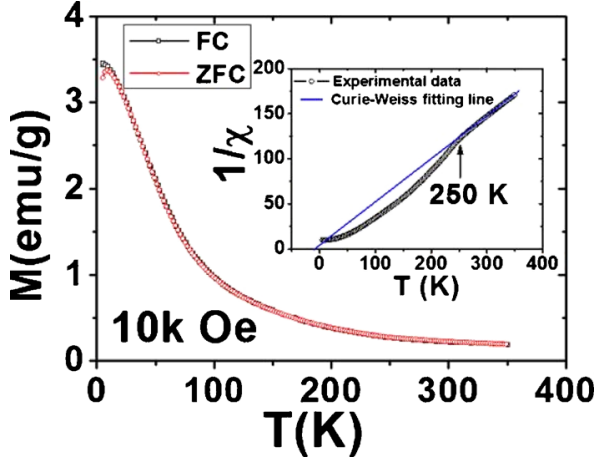

FIG. 6. (Color online) ZFC and FC magnetizations of LBFM ceramic recorded at $10 \mathrm{kOe}$, inset is the plot of $1 / \chi$ vs temperature fitted to CurieWeiss law.

FC magnetizations of LBFM ceramic were recorded at 10 kOe as shown in Fig. 6. The inset figure shows the reverse susceptibility $(1 / \chi)$ of LBFM ceramic. Above the spin-glass behavior, the antiferromagnetic transition at $250 \mathrm{~K}$ was observed. $\mathrm{Bi}$ et $a l^{10}$ has calculated three structures of $\mathrm{Bi}_{2} \mathrm{FeMnO}_{6}$. For LBFM, we believe it exhibits phase transition although the uncertainties of the exact phases. The data from 350 to $250 \mathrm{~K}$ were fitted to the Curie-Weiss law. The parameters of Curie constant (C), effective paramagnetic moment $\left(\mu_{\text {eff }}\right)$, and Weiss constant $(\theta)$ were determined by the Curie-Weiss fitting to be 1.92 emu $\mathrm{K} \mathrm{mol}^{-1}$ f.u., $3.91 \mu_{\mathrm{B}}$, and $-9 \mathrm{~K}$, respectively.

As shown in Fig. 7(a), from the real part of ac susceptibility measured at different frequencies, we can see a peak
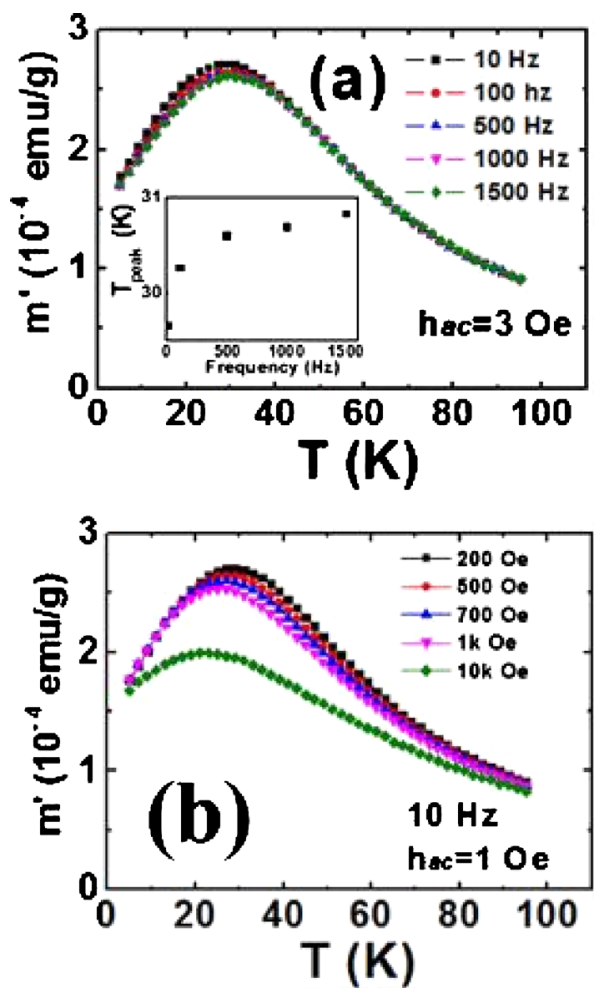

FIG. 7. (Color online) (a) Temperature dependence of the ac magnetization obtained with different frequencies, inset figure shows the shift in peak temperature $\left(\mathrm{T}_{\text {peak }}\right)$ with frequency; (b) the ac magnetization vs temperature under different applied static field and $10 \mathrm{~Hz}$ fixed frequency and $h_{a c}$ $=1$ Oe. which changes very little with different frequency (inset figure). The possible shift to high frequency is not clear, that is partially due to the less points measured around the peak. However, it can be found that all the curves on the left side shift clearly to high frequency, while, the curves on the right side of the peak remain the same as that of the lowest frequency. Figure 7(b) shows the real part of ac magnetization versus temperature at $10 \mathrm{~Hz}$ fixed frequency and $\mathrm{h}_{\mathrm{ac}}=1$ Oe. Different static fields of $200 \mathrm{Oe}, 500 \mathrm{Oe}, 700 \mathrm{Oe}, 1 \mathrm{kOe}$, and $10 \mathrm{kOe}$ were applied. It clearly shows that the values of $\mathrm{m}^{\prime}$ decreased and the peak moved to low temperature when the field increases. This indicates that the possible spin glass state takes place at low temperature when increasing magnetic field. In another word, the magnetic field pushes the spin glass to occur at low temperature.

There are hysteresis loops below the spin glass transition temperature as show in Fig. 4 and a small negative $\theta$ value was obtained through the Curie-Weiss fitting in Fig. 6 inset. Therefore, similar to $\mathrm{BiFeO}_{3}$ (Ref. 21) there are at least three clear evidences for spin-glass behavior in LBFM ceramic: first, there is a large difference between FC and ZFC at low temperature; second, the cusp was observed in ZFC curves; third, the peak temperature is dependent upon the frequency and applied field. Based on these data, we propose that the spin glass state probably arises from a weak ferromagnetic or ferrimagnetic transition around the $T_{g}$, or from the competition between ferromagnetic and antiferromagnetic transition.

\section{SUMMARY}

LBFM ceramic and film which was deposited on (100) $\mathrm{SrTiO}_{3}$ by PLD method are first studied. The LBFM film is antiferromagnetic or weak ferrimagnetic (ferromagnetic plus antiferromagnetic configuration) at room temperature. However, further studies should be carried out on the determination of antiferromagnetic spin configuration using neutron diffraction at temperatures above room temperature. Because of the substrate influence, whether the phenomena in the LBFM film can be classified as a classical spin-glass still remains open. For the LBFM ceramic, all the observations indicate that the spin glass state is present at low temperature. While compared to LBFM film, the ceramic shows a $T_{N}$ below room temperature. The different properties between film and ceramic are due to the delicate effects like structure, strain, valence, spin, charge and so on.

\section{ACKNOWLEDGMENTS}

The authors gratefully acknowledge Dr. Shigeki Nimori, Dr. Hideaki Kitazawa, and Dr. Wenhong Wang of NIMS for the valuable discussions about magnetic properties. Part of this work was supported by grants from JSPS and ARC under the Japan-Australia Research Cooperative Program, Grant-in-Aid for JSPS Fellows (21-09608) and the Australian Research Council.

${ }^{1}$ R. Ramesh and N. A. Spaldin, Nature Mater. 6, 21 (2007).

${ }^{2}$ N. S. Rogado, J. Li, A. W. Sleight, and M. A. Subramanian, Adv. Mater. 17, 2225 (2005).

${ }^{3}$ M. Azuma, K. Takata, T. Saito, S. Ishiwata, Y. Shimakawa, and M. Takano, J. Am. Chem. Soc. 127, 8889 (2005). 
${ }^{4}$ G. Blasse, J. Phys. Chem. Solids 26, 1969 (1965)

${ }^{5}$ R. I. Dass, J. Q. Yan, and J. B. Goodenough, Phys. Rev. B 68, 064415 (2003).

${ }^{6}$ R. I. Dass and J. B. Goodenough, Phys. Rev. B 67, 014401 (2003).

${ }^{7}$ K. De, M. Thakur, A. Manna, and S. Giri, J. Appl. Phys. 99, 013908 (2006).

${ }^{8}$ Y. Tokura and N. Nagaosa, Science 288, 462 (2000).

${ }^{9}$ E. Dagotto, T. Hotta, and A. Moreo, Phys. Rep. 344, 1 (2001).

${ }^{10}$ L. Bi, A. R. Taussig, H. S. Kim, L. Wang, G. F. Dionne, D. Bono, K Persson, G. Ceder, and C. A. Ross, Phys. Rev. B 78, 104106 (2008).

${ }^{11}$ H. Zhao, H. Kimura, Z. X. Cheng, X. L. Wang, K. Ozawa, and T. Nishida, Phys. Status Solidi (RRL) (in press).

${ }^{12}$ S. Fujino, M. Murakami, S. H. Lim, L. G. Salamanca-Riba, M. Wuttig, and I. Takeuchi, J. Appl. Phys. 101, 013903 (2007).

${ }^{13}$ M. Gajek, M. Bibes, S. Fusil, K. Bouzehouane, J. Fontcuberta, A. Barthelemy, and A. Fert, Nature Mater. 6, 296 (2007).
${ }^{14}$ M. Gajek, M. Bibes, F. Wyczisk, M. Varela, J. Fontcuberta, and A. Barthelemy, Phys. Rev. B 75, 174417 (2007).

${ }^{15}$ Z. X. Cheng, A. H. Li, X. L. Wang, S. X. Dou, K. Ozawa, and H. Kimura, J. Appl. Phys. 103, 07E507 (2008).

${ }^{16}$ E. Langenberg, M. Varela, M. V. Garcia-Cuenca, C. Ferrater, M. C. Polo, I. Fina, L. Fabrega, F. Sanchez, and J. Fontcuberta, J. Magn. Magn. Mater. 321, 1748 (2009).

${ }^{17}$ K. De, R. Ray, R. N. Panda, S. Giri, H. Nakamura, and T. Kohara, J. Magn. Magn. Mater. 288, 339 (2005).

${ }^{18}$ W. Tong, B. Zhang, S. Tan, and Y. Zhang, Phys. Rev. B 70, 014422 (2004).

${ }^{19}$ M. N. Iliev, M. V. Abrashev, A. P. Litvinchuk, V. G. Hadjiev, H. Guo, and A. Gupta, Phys. Rev. B 75, 104118 (2007).

${ }^{20}$ G. F. Dionne, J. Appl. Phys. 101, 09C509 (2007).

${ }^{21}$ G. Catalan and J. F. Scott, Adv. Mater. 21, 2463 (2009). 